\title{
DECOMPOSIÇÃO DE RESÍDUOS INDUSTRIAIS NO SOLO
}

\author{
'Marcelo de Paula Segatto, 'Robson Andreazza, ' Leandro Bortolon, \\ 'Viviane Pereira Santos, 'Clesio Gianello, \\ 'Flávio A.O. Camargo \\ 'Universidade Federal do Rio Grande do Sul; Porto Alegre, RS \\ ${ }^{2}$ EMBRAPA \\ e-mail: robsonandreazza@yahoo.com.br
}

\section{Resumo}

O descarte de resíduos no solo é uma alternativa viável para reduzir o impacto ambiental de muitos resíduos industriais, além de esses materiais serem utilizados como importante fonte de nutrientes para as plantas. Assim, o objetivo deste trabalho foi estimar a taxa de decomposição de quatro resíduos industriais aplicados em dois diferentes solos (Latossolo e Argissolo) utilizados a este propósito. Os resíduos das estações de tratamento de indústrias foram: Cervejarias Kaiser (CERV), Laticínios Dália (LAT) Satipel, chapas de madeira aglomerada (AGL) e tecelagem Sultêxtil (TEXT). Foi avaliada a decomposição desses resíduos através da atividade microbiana nos dois solos pela evolução de C- $\mathrm{CO}_{2}$ e mineralização de N. Os resíduos orgânicos avaliados podem ser usados em solo agrícola, entretanto deve ser realizada adubação complementar com $\mathrm{N}$ mineral. A taxa de decomposição dos resíduos nos dois solos avaliados, determinada pela liberação de $\mathrm{C}-\mathrm{CO}_{2}$ e pelo acúmulo de $\mathrm{N}$ mineral no solo, aumentou na ordem: TEXT>LAT>CERV>AGL.

Palavras-chave: resíduos orgânicos; atividade microbiana; carbono; nitrogênio. 


\section{Abstract}

Waste disposal in soils is an efficient alternative to reduce environmental negative impact of industrial wastes and can be used as an important nutrients source for the plants. Thus, the aim of this study was to evaluate the decomposition rate of four industrial wastes applied in two different soils (Oxisol and Ultisol). The industrial wastes of treatment station were: (brewery - CERV; dairy products - LAT; plywood - AGL and textile - TEXT). It was evaluated the decomposition of the doses of these wastes through the microbial activity and $\mathrm{N}$ mineralization. The organic wastes evaluated can be used in agricultural soils, although, should be used nitrogen as additional fertilization. The rate of the decomposition of the wastes in the both evaluated soils, for the $\mathrm{C}-\mathrm{CO}_{2}$ release and for the $\mathrm{N}$ accumulation in the soils, increased in the following order: TEXT >LAT>CERV>AGL.

Keywords: organic residues; microbial activity; carbon; nitrogen.

\section{Introdução}

A crescente produção mundial vem aumentando a quantidade de resíduos industriais (REBAH et al., 2007) e assim aumentando a necessidade de viabilizar uma solução para o tratamento e ou utilização desses resíduos (WEI e HUANG, 2001; STIJEPOVIC e LINKE, 2011). A disposição de resíduos orgânicos em solos agrícolas vem sendo utilizada de forma crescente em nível mundial, sendo uma alternativa economicamente viável e, se bem conduzida, ambientalmente correta (SMITH, 2009).

Por suas características químicas e biológicas, o solo propicia as condições necessárias para a biodegradação de resíduos orgânicos. Além disso, o material orgânico pode disponibilizar nutrientes, como o nitrogênio, o fósforo e o potássio para as plantas e microrganismos, além de melhorar os atributos químicos e físicos do solo pela adição de matéria orgânica (MOREIRA e SIQUEIRA, 2006). Entretanto, dependendo da dose adicionada, pode haver efeitos prejudiciais ao ambiente (LORENZ e LAL, 2009). Dessa forma, a taxa de decomposição dos materiais é uma maneira de se verificar o potencial dos resíduos orgânicos aplicados ao solo sem causar efeitos prejudiciais ao ambiente, como a elevada emissão de gases à atmosfera. A decomposição de resíduos orgânicos adicionados ao solo pode ser avaliada pela determinação da atividade microbiana por meio da quantificação do $\mathrm{CO}_{2}$ liberado pela respiração resultante da atividade dos microorganismos (STOTZKY, 1965). Atualmente, existem muitos trabalhos sobre a adição de resíduos de origem 
animal e urbano, como, por exemplo, lodo de esgoto e composto de lixo no solo, porém pouco se sabe sobre a adição de resíduos provenientes unicamente da atividade industrial e estudos são necessários para gerar informações sobre os efeitos da disposição desses resíduos nos solos.

Os resíduos orgânicos são constituídos por compostos com diferentes graus de biodegradabilidade. Os compostos mais facilmente degradáveis são os açúcares, o amido e as proteínas; em um estágio intermediário, situam-se a celulose e a hemicelulose, sendo as substâncias fenólicas e gorduras as mais resistentes à decomposição (MAISTO et al., 2010).

A relação C:N é um importante parâmetro para a predição da decomposição do resíduo, indicando as possíveis transformações e disponibilidade do nitrogênio. $\mathrm{O}$ processo de mineralização do $\mathrm{N}$ é promovido quando a relação C:N é de aproximadamente 20: (LORENZ e LAL, 2009). Os resíduos de baixa relação C:N e de fácil degradabilidade podem estimular a decomposição do carbono presente no solo, o que é denominado de "efeito priming". Este efeito foi constatado por Hernandez et al. (1988), que determinaram teor de carbono orgânico maior em solo sem adição de resíduo do que naqueles em que houve adição, após seis meses da aplicação. A incubação de solo com resíduos orgânicos em laboratório é um procedimento seguro, pois possibilita avaliações do $\mathrm{CO}_{2}$ evoluído em ambiente controlado, em tempo relativamente curto (CERRI et al., 1994; MAISTO et al., 2010). Assim, este trabalho teve como objetivo estimar a taxa de decomposição de quatro resíduos industriais aplicados em dois tipos de solos utilizados para esse propósito.

\section{Material e métodos}

Para a avaliação dos efeitos da utilização dos resíduos orgânicos no solo, foram utilizados dois tipos de solos sob vegetação nativa, Latossolo Vermelho distroférrico nitossólico (LVd) e Argissolo Vermelho distrófico arênico (PVd) (EMBRAPA, 2006). O solo foi coletado na profundidade de zero a $20 \mathrm{~cm}$. Após a coleta e homogeneização dos solos, foi retirada uma subamostra para as análises laboratoriais. $\mathrm{O}$ solo foi coletado na profundidade de zero a $20 \mathrm{~cm}$ e, após a coleta e homogeneizações dos solos, foram retiradas as amostras para análise. Estas foram secas à $45^{\circ} \mathrm{C}$, moídas em moinho de martelo e então determinadas, conforme metodologia descrita por Tedesco et al. (1995), os seguintes atributos: pH em água (relação 1:1); índice SMP; matéria orgânica; fósforo e potássio disponíveis; sódio, cálcio, magnésio, alumínio e manganês trocáveis; enxofre extraível; zinco, cobre e boro extraíveis (Tabela 1). 
Os resíduos orgânicos utilizados foram obtidos das estações de tratamento de efluentes (ETEs) dos seguintes estabelecimentos: Cervejaria Kaiser (15 t mês $\mathrm{s}^{-1}$ e lodo em tanque anaeróbio e aeróbio), localizada em Gravataí - RS; Laticínios Dália (160 t mês ${ }^{-1}$ de lodo em duas lagoas de estabilização aeróbias), situada em Arroio do Meio - RS; tecelagem Sultêxtil (1,5 a 2,0 t mês $\mathrm{s}^{-1}$ de lodo ativado - tanque de aeração) localizada em Caxias do Sul - RS; e Satipel, indústria de madeira aglomerada $\left(1,0 \mathrm{~m}^{3} \mathrm{mês}^{-1}\right.$, lodo primário), localizada no município de Taquari - RS. Amostras representativas de cada material foram coletadas para realização da análise química (TEDESCO et al., 1995), sendo os resultados apresentados na Tabela 2.

A liberação de $\mathrm{C}_{-} \mathrm{CO}_{2}$ foi determinada em condição de laboratório por um período de 88 dias após a adição dos resíduos orgânicos. Porções de $200 \mathrm{~g}$ de solo foram misturadas com os materiais orgânicos, em duas doses 1 e 2, equivalentes a 100 e $300 \mathrm{~kg} \mathrm{ha}^{-1}$ de $\mathrm{N}$ total, respectivamente. Foram incluídos também um tratamento com adubação mineral, a mesma dose de nitrogênio na forma de ureia e um tratamento testemunha somente com solo, além da prova em branco, com duas repetições. O fósforo e o potássio foram aplicados em todos os tratamentos, à exceção da testemunha, nas quantidades de: 240 e $180 \mathrm{~kg} \mathrm{ha}^{-1}$ de $\mathrm{P}_{2} \mathrm{O}_{5}$ (superfosfato triplo) e $\mathrm{K}_{2} \mathrm{O}$ (KCl), respectivamente, no solo PVd; 180 e $40 \mathrm{~kg} \mathrm{ha}^{-1}$ de $\mathrm{P}_{2} \mathrm{O}_{5}$ (superfosfato triplo) e $\mathrm{K}_{2} \mathrm{O}(\mathrm{KCl})$, respectivamente, no solo LVd (SBCS/NRS, 2004). O pH do solo foi corrigido para 6,o com base no valor neutralizante de cada resíduo, complementado com a mistura de $\mathrm{CaCO}_{3}+\mathrm{MgCO}_{3}$ (3:1). Os resíduos secos e moídos foram misturados ao solo, adicionando-se a seguir água destilada até atingir $80 \%$ da capacidade de campo, sendo mantida a mesma umidade até o fim do experimento, pela complementação da variação da massa dos tratamentos.

A contribuição do "efeito priming" para o total de $\mathrm{CO}_{2}$ emitido só é possível de ser quantificada pela utilização de isótopos. Como neste caso não foi feita a marcação do carbono do material orgânico, a quantificação do $\mathrm{CO}_{2}$ devido à incorporação do substrato orgânico foi obtida pela diferença entre o $\mathrm{CO}_{2}$ determinado no solo em que foi adicionado resíduo menos o $\mathrm{CO}_{2}$ determinado em um tratamento com solo sem adição de resíduo (testemunha). 
Tabela 1. Caracterização físico-química da camada superficial $(0-20 \mathrm{~cm})$ do Latossolo VermeIho distroférrico nitossólico (LVd) e Argissolo Vermelho distrófico arênico (PVd).

\begin{tabular}{|c|c|c|}
\hline \multirow{2}{*}{ Atributos } & \multicolumn{2}{|c|}{ Solos } \\
\hline & LVd & PVd \\
\hline pH em água & 4,9 & 4,5 \\
\hline Índice SMP & 5,6 & 6,2 \\
\hline P disponível (mg dm³) & 5,0 & 2,8 \\
\hline $\mathrm{K}$ disponível (mg dm³) & 126 & 18 \\
\hline Matéria orgânica ( $\left.\mathrm{g} \mathrm{dm}^{-3}\right)$ & 24 & 9 \\
\hline Al trocável $\left(\mathrm{cmol}_{\mathrm{c}} \mathrm{dm}^{-3}\right)$ & 0,4 & 1,5 \\
\hline Ca trocável $\left(\mathrm{cmol}_{\mathrm{c}} \mathrm{dm}^{-3}\right)$ & 3,1 & 0,3 \\
\hline $\mathrm{Mg}$ trocável $\left(\mathrm{cmol}_{\mathrm{c}} \mathrm{dm}^{-3}\right)$ & 0,9 & 0,2 \\
\hline $\mathrm{Al}+\mathrm{H}\left(\mathrm{cmol}_{\mathrm{c}} \mathrm{dm}^{-3}\right)$ & 5,1 & 3,0 \\
\hline CTC $\left(\mathrm{cmol}_{c} \mathrm{dm}^{-3}\right)$ & 9,6 & 3,5 \\
\hline Saturação por bases (\%) & 45 & 15 \\
\hline Saturação por Al (\%) & 4,2 & 42,3 \\
\hline $\mathrm{S}\left(\mathrm{mg} \mathrm{dm^{-3 } )}\right.$ & 14 & 17 \\
\hline $\mathrm{Zn}\left(\mathrm{mg} \mathrm{dm}^{-3}\right)$ & 5,5 & 0,3 \\
\hline $\mathrm{Cu}\left(\mathrm{mg} \mathrm{dm}^{-3}\right)$ & 5,2 & 0,9 \\
\hline$B\left(\mathrm{mg} \mathrm{dm}^{-3}\right)$ & 0,6 & 0,7 \\
\hline $\mathrm{Mn}\left(\mathrm{mg} \mathrm{dm} \mathrm{m}^{-3}\right)$ & 97 & 14 \\
\hline Argila $\left(\mathrm{g} \mathrm{dm}^{-3}\right)$ & 300 & 130 \\
\hline Capacidade de campo $(\%) 2$ & 22,8 & 15,2 \\
\hline
\end{tabular}

' Conforme metodologia descrita por Tedesco et al. (1995).

${ }^{2}$ Método da proveta, umidade gravimétrica. 


\section{ciênciarnatura UFSM, 34(1)}

Tabela 2. Características físico-químicas dos resíduos utilizados: Laticínios Dália (LAT), Cervejarias Kaiser (CERV), Satipel, chapas de madeira aglomerada (ACL) e tecelagem Sultêxtil (TEXT).

\begin{tabular}{|c|c|c|c|c|}
\hline \multirow{2}{*}{ Características" } & \multicolumn{4}{|c|}{ Resíduos } \\
\hline & LAT & CERV & $A G L$ & TEXT \\
\hline Teor de sólidos ( $\mathrm{g} \mathrm{kg}^{-1}$ ) & 10 & 130 & 90 & 870 \\
\hline $\mathrm{pH}$ em água & 7,2 & 7,0 & 7,6 & 6,8 \\
\hline Carbono orgânico $\left(\mathrm{g} \mathrm{kg}^{-1}\right)$ & 340 & 120 & 100 & 550 \\
\hline Nitrogênio total $\left(\mathrm{g} \mathrm{kg}^{-1}\right)$ & 58 & 17 & 186 & 50 \\
\hline Relação C/N & 5,9 & 7,1 & 0,5 & 11,0 \\
\hline $\mathrm{NH}_{4}+\left(\mathrm{mg} \mathrm{kg}^{-1}\right)$ & 1100 & 792 & 1700 & 1800 \\
\hline $\mathrm{NO}_{3}^{-}+\mathrm{NO}_{2}^{-}\left(\mathrm{mg} \mathrm{kg}^{-1}\right)$ & 60 & 22 & 1200 & 103 \\
\hline Fósforo total (g kg-1) & 26 & 7,8 & 0,3 & 8,0 \\
\hline Potássio total $\left(\mathrm{g} \mathrm{kg}^{-1}\right)$ & 5,3 & 1,3 & 0,3 & 1,6 \\
\hline Cálcio (g kg-1) & 22,0 & 5,4 & 12,0 & 7,0 \\
\hline Magnésio $\left(\mathrm{g} \mathrm{kg}^{-1}\right)$ & 3,5 & 0,8 & 1,2 & 1,9 \\
\hline Enxofre ( $\mathrm{g} \mathrm{kg}^{-1}$ ) & 8,6 & 3,5 & 18,0 & 14,0 \\
\hline Cobre $\left(\mathrm{mg} \mathrm{kg}^{-1}\right)$ & 154 & 206 & 9,2 & 605 \\
\hline Zinco $\left(\mathrm{g} \mathrm{kg}^{-1}\right)$ & 0,3 & 1,7 & $<0,1$ & 3,0 \\
\hline Ferro $\left(\mathrm{g} \mathrm{kg}^{-1}\right)$ & 3,8 & 10,0 & 0,8 & 6,2 \\
\hline Manganês (mg kg-1) & 241 & 119 & 28 & 113 \\
\hline Sódio $\left(\mathrm{g} \mathrm{kg}^{-1}\right)$ & 35 & 2,5 & 4,0 & 3,8 \\
\hline Cromo $\left(\mathrm{mg} \mathrm{kg}^{-1}\right)$ & 38 & 153 & 12 & 102 \\
\hline Cádmio (mg kg-1) & 0,96 & 1,9 & 2,3 & 0,57 \\
\hline Níquel (mg kg-1) & 14 & 21 & 7,8 & 38 \\
\hline Chumbo (mg kg-1) & 22 & 80 & 14 & 18 \\
\hline Mercúrio $\left(\mu \mathrm{g} \mathrm{kg}^{-1}\right)$ & 52 & 362 & 102 & 532 \\
\hline Valor de neutralização $\left(\mathrm{g} \mathrm{kg}^{-1}\right)$ & 84 & 27 & 16 & 16 \\
\hline
\end{tabular}

* Determinações feitas no material seco a $75^{\circ} \mathrm{C}$, à exceção do $\mathrm{pH}$.

A mistura foi acondicionada em frascos de vidro com capacidade de $1000 \mathrm{~mL}$ e sobre a superfície do solo de cada frasco de incubação foi colocado um suporte de inox contendo um frasco de polietileno $(50 \mathrm{~mL}) \mathrm{com}$ $20 \mathrm{~mL}$ de solução de $\mathrm{NaOH}$ de concentração $0,5 \mathrm{~mol} \mathrm{~L}^{-1}$, sendo então o frasco hermeticamente fechado. Em intervalos variáveis, os copos contendo 
$\mathrm{NaOH}$ foram retirados dos frascos para a determinação do $\mathrm{CO}_{2}$ liberado, adicionando-se um $\mathrm{mL}$ de uma solução de $\mathrm{BaCl}_{2}\left(2,5 \mathrm{~g} \mathrm{~L}^{-1}\right)$ e três gotas de fenolftaleína $\left(0,5 \mathrm{~g} \mathrm{~L}^{-1}\right)$; em seguida o excesso de $\mathrm{NaOH}$, ou seja, a fração não consumida na reação com $\mathrm{CO}_{2}$ foi titulada com solução padronizada de $\mathrm{HCl}\left(0,5 \mathrm{~mol} \mathrm{~L}^{-1}\right)$. As determinações foram feitas aos 1, 3, 6, 13, 20, 26, 32, $39,46,53,60,67,74,81$ e 88 dias após o início da incubação. A liberação de $\mathrm{C}-\mathrm{CO}_{2}$, em mg de C-CO $\mathrm{kg}^{-1}$ de solo, foi calculada pela seguinte fórmula $\mathrm{C}-\mathrm{CO}_{2}\left(\mathrm{mg} \mathrm{kg}^{-1}\right)=(\mathrm{VB}-\mathrm{VA})$. M . E . 5, em que: VB = volume de solução de $\mathrm{HCl}$ gasto na titulação da prova em branco $(\mathrm{mL}) ; \mathrm{VA}=$ volume de solução de $\mathrm{HCl}$ gasto na titulação da amostra $(\mathrm{mL}) ; \mathrm{M}=$ concentração molar da solução de $\mathrm{HCl}\left(0,5 \mathrm{~mol} \mathrm{~L}^{-1}\right) ; \mathrm{E}$ = peso equivalente grama do carbono.

No final da incubação, foi retirada uma amostra de solo de cada unidade experimental e determinados, na amostra úmida, o pH e os teores de nitrogênio inorgânico $\left(\mathrm{NH}^{4+}\right.$ e $\left.\mathrm{NO}^{3-}+\mathrm{NO}^{2-}\right)$, conforme metodologia descrita por Tedesco et al. (1995). A taxa de mineralização do $\mathrm{N}$, para dose $300 \mathrm{~kg} \mathrm{ha}^{-1}$ de $\mathrm{N}$ no resíduo foi obtida pela equação: TMN $=\left[\left(\mathrm{N}_{\text {trat }}-\mathrm{N}_{\text {test }}\right) / \mathrm{N}_{\text {adic }}\right] \times 100$, em que: TMN = taxa de mineralização do $\mathrm{N}$ do resíduo(\%); Ntrat = teor de $\mathrm{N}$ mineral no solo no tratamento com resíduo $\left(\mathrm{mg} \mathrm{kg}^{-1}\right) ; \mathrm{N}_{\text {test }}=$ teor de $\mathrm{N}$ mineral no solo no tratamento testemunha $\left(\mathrm{mg} \mathrm{kg}^{-1}\right) ; \mathrm{N}_{\text {adic }}=$ quantidade de $\mathrm{N}$ adicionada pelo resíduo $\left(\mathrm{mg} \mathrm{kg} \mathrm{kg}^{-1}\right)$.

A análise dos dados foi realizada pela análise da variância individual e conjunta dos dados, o teste de significância foi realizado pelo teste $\mathrm{F}$ e, quando significativo, foi realizada a comparação múltipla de médias pelo teste de Tukey, ao nível de 5\% de probabilidade.

\section{Resultados e discussão}

As quantidades cumulativas de $\mathrm{CO}_{2}$ evoluído durante o período de incubação são apresentadas nas Figuras 1 e 2. As quantidades emitidas até os 32 dias de incubação variaram entre 56 a $70 \%$ do carbono total produzido em todo o período de incubação, demonstrando que a maior atividade dos microorganismos do solo ocorre nos primeiros dias após a adição de materiais orgânicos no solo (METZGER e YARON, 1987; MALIK et al., 2008). Esse fato indica que estudos com curta duração podem fornecer informações úteis a respeito da decomposição de resíduos no solo. Após este período inicial, houve um declínio na atividade microbiana do solo. Os valores determinados nos tratamentos com adubação mineral ou adição de resíduos orgânicos foram 
sempre superiores ao tratamento testemunha, nos dois solos (Figuras 1 e 2). Isto, devido à maior quantidade de nutrientes ou compostos orgânicos (fonte energética) disponíveis aos microrganismos, onde são necessários à multiplicação microbiana (MOREIRA e SIQUEIRA, 2006). A adição de resíduos no solo pode alterar a atividade da microbiota, devido ao tipo da composição química da fração orgânica do resíduo, influenciando na decomposição da matéria orgânica do solo (HERNANDEZ et al., 1988).
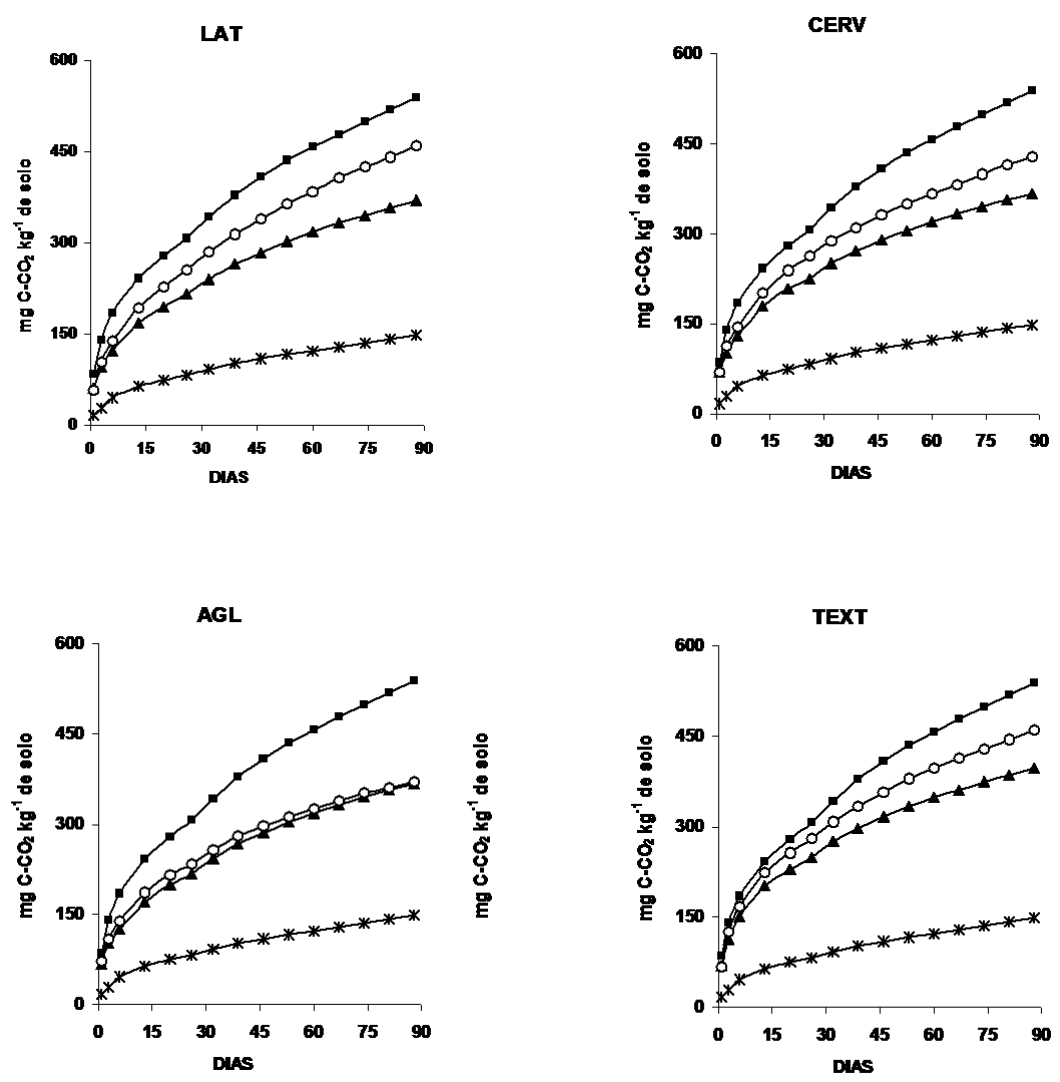

* Test. $\rightarrow$ Dose $1 \rightarrow$ Dose $2 \rightarrow$ NPK

Figura 1. Quantidade cumulativa de carbono $\left(\mathrm{C}-\mathrm{CO}_{2}\right)$ evoluído de Argissolo Vermelho distrófico arênico (PVd) com adição de quatro resíduos orgânicos: Laticínios Dália (LAT) Satipel, Cervejarias Kaiser (CERV), chapas de madeira aglomerada (AGL) e tecelagem Sultêxtil (TEXT). 

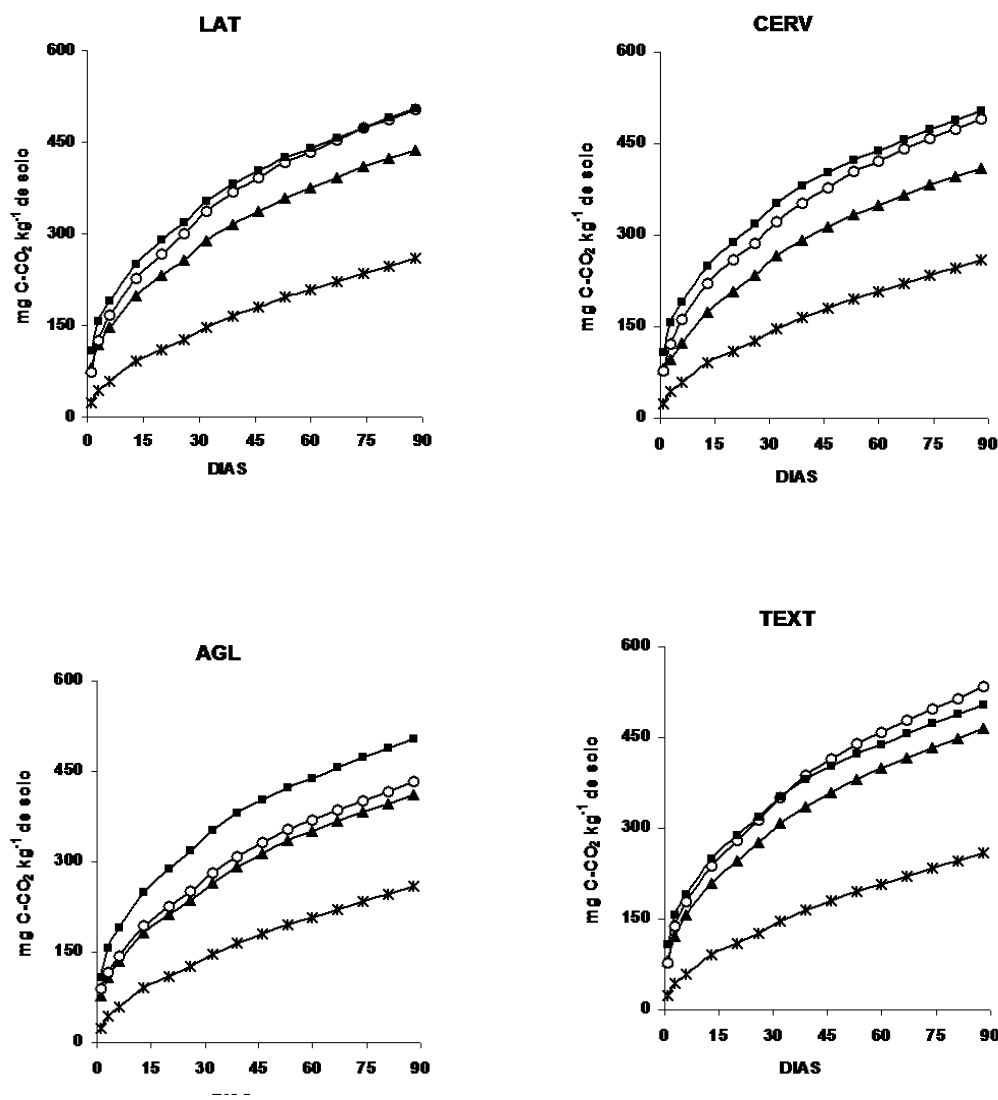

$$
\text { *-Test. } \leftarrow \text { Dose } 1 \rightarrow \text { Dose } 2 \rightarrow \text { NPK }
$$

Figura 2. Quantidade cumulativa de carbono (C-CO2) evoluído de Latossolo Vermelho distroférrico nitossólico (LVd) com a adição de quatro resíduos orgânicos: Laticínios Dália (LAT) Satipel, Cervejarias Kaiser (CERV), chapas de madeira aglomerada (AGL) e tecelagem Sultêxtil (TEXT).

Não foi observada diferença significativa entre as quantidades de $\mathrm{C}-\mathrm{CO}_{2}$ evoluído para as doses de resíduo adicionadas no solo PVd (Tabela 3). No solo LVd, entretanto, a quantidade de $\mathrm{C}_{-} \mathrm{CO}_{2}$ evoluído aumentou com o acréscimo das quantidades de resíduo adicionadas, à exceção do resíduo AGL. O efeito diferenciado em cada solo pode estar relacionado com a capacidade de utilização de substratos orgânicos pela microbiota do solo, pois, em geral, solos com alto teor de matéria orgânica e argila apresentam 
maior quantidade e diversidade microbiana do que solos com baixos teores de matéria orgânica (ØVREÅS e TORSVIK, 1998; MALIK et al., 2008). No Argissolo, as maiores quantidades de $\mathrm{C}-\mathrm{CO}_{2}$ emitidos foram determinadas no tratamento com adubação mineral completa, sem adição de resíduos (Tabela 3). A diferença da atividade microbiana entre os tratamentos com composto orgânico e adubação mineral em relação à testemunha foi maior no solo PVd (Tabela 3), devido à sua baixa fertilidade e menor suprimento de nutrientes para a microbiota, principalmente nitrogênio e fósforo (Tabela ı).

Tabela 3. Quantidade cumulativa de carbono (mg de C-CO2 kg-1 de solo) evoluído durante a incubação no Latossolo Vermelho distroférrico nitossólico (LVd) e Argissolo Vermelho distrófico arênico (PVd).

\begin{tabular}{|c|c|c|c|c|c|c|c|c|c|}
\hline \multirow{2}{*}{ Tratamento } & \multirow{2}{*}{ Dose } & \multicolumn{8}{|c|}{ Dias de incubação } \\
\hline & & \multicolumn{2}{|c|}{13} & \multicolumn{2}{|c|}{32} & \multicolumn{2}{|c|}{60} & \multicolumn{2}{|c|}{88} \\
\hline \multicolumn{10}{|c|}{ - } \\
\hline Testemunha & - & $91^{*}$ & $\mathrm{~d}$ & 146 & $\mathrm{e}$ & 207 & $\mathrm{e}$ & 259 & $\mathrm{e}$ \\
\hline NPK & - & 249 & $\mathrm{a}$ & 353 & a & 439 & $a b$ & 505 & $a b$ \\
\hline$A G L$ & 1 & 182 & c & 264 & $d$ & 352 & $d$ & 411 & $d$ \\
\hline$A G L$ & 2 & 193 & bc & 282 & $\mathrm{~cd}$ & 370 & $\mathrm{~cd}$ & 433 & $\mathrm{~cd}$ \\
\hline CERV & 1 & 175 & c & 267 & $d$ & 349 & $d$ & 410 & $d$ \\
\hline CERV & 2 & 221 & $a b$ & 323 & $a b$ & 422 & $a b$ & 490 & $a b$ \\
\hline LAT & 1 & 198 & bc & 288 & $\mathrm{~cd}$ & 375 & $\mathrm{~cd}$ & 437 & $\mathrm{~cd}$ \\
\hline LAT & 2 & 226 & $a b$ & 336 & $a b$ & 433 & $a b$ & 502 & $a b$ \\
\hline TEXT & 1 & 210 & bc & 309 & bc & 400 & bc & 466 & bc \\
\hline TEXT & 2 & 238 & $\mathrm{a}$ & 351 & $\mathrm{a}$ & 459 & $\mathrm{a}$ & 534 & a \\
\hline \multicolumn{10}{|c|}{-ap-1-1 } \\
\hline Testemunha & - & 64 & $\mathrm{~d}$ & 92 & $\mathrm{~d}$ & 122 & $\mathrm{~d}$ & 149 & $\mathrm{~d}$ \\
\hline NPK & - & 242 & $a$ & 343 & $a$ & 458 & $a$ & 538 & a \\
\hline$A G L$ & - & 179 & c & 251 & c & 322 & c & 370 & c \\
\hline CERV & - & 190 & c & 270 & c & 343 & bc & 398 & bc \\
\hline LAT & - & 181 & c & 263 & c & 351 & $b$ & 415 & bc \\
\hline TEXT & - & 214 & $b$ & 291 & $b$ & 373 & $b$ & 429 & $b$ \\
\hline
\end{tabular}


Em geral, a adubação mineral proporcionou maiores quantidades de C- $\mathrm{CO}_{2}$ emitidos nos dois solos em estudo (Figuras 1 e 2), contudo, com a adição da Dose 2 do resíduo de indústria têxtil no Latossolo, a emissão de $\mathrm{CO}_{2}$ foi superior a todos os tratamentos (Figura 2). As pequenas quantidades de $\mathrm{C}-\mathrm{CO}_{2}$ emitidos podem ser devido à menor disponibilidade de nitrogênio mineral no solo (Tabela 4). Embora haja a aplicação de $100 \mathrm{~kg} \mathrm{ha}^{-1} \mathrm{de} \mathrm{N}$ nessa taxa de aplicação de resíduos, a mineralização do N orgânico destes foi insuficiente para o suprimento da microbiota do solo. Apesar de os resíduos apresentarem teores elevados de $\mathrm{N}$ total (Tabela 2) e baixa relação C:N, eles são materiais já estabilizados nas estações de tratamento, onde sua mineralização é muita lenta. O aumento das quantidades de resíduo adicionado, suficiente para suprir $300 \mathrm{~kg} \mathrm{ha}^{-1}$ de $\mathrm{N}$, provocou, na maior parte dos casos, pequeno aumento nas quantidades de $\mathrm{C}_{-} \mathrm{CO}_{2}$ emitidos (Figuras 1 e 2), sendo que, no caso do AGL, este aumento foi menor do que nos demais tratamentos, provavelmente pelo material ser composto de uréia-formol-melanina (LORENZ e LAL, 2009).

Os teores de $\mathrm{N}$ mineral acumulado ao final da incubação aumentaram significativamente para o resíduo TEXT nos dois solos, com o acréscimo das doses de resíduo adicionado (Tabela 4). O aumento das quantidades de $\mathrm{C}-\mathrm{CO}_{2}$ mineralizado com o acréscimo das doses de resíduos aplicadas indica que estes afetam positivamente o crescimento da microbiota do solo. A mineralização do nitrogênio nos tratamentos com incorporação de resíduo ao solo pode ser avaliada pelos teores de nitrogênio mineral no solo $\left(\mathrm{NH}^{4+}\right.$ e $\mathrm{NO}^{3}+\mathrm{NO}^{2}$ ) ao final do experimento.

Os resíduos apresentaram as seguintes taxas de mineralização média ao final da incubação para os dois solos: TEXT $=37,6 \%$; LAT $=24,3 \%$; CERV $=18,2 \%$ e AGL $=16,4 \%$, calculadas para a dose equivalente a 300 $\mathrm{kg} \mathrm{ha}^{-1}$ de $\mathrm{N}$ no resíduo. Essas taxas foram maiores no solo PVd do que no LVd, para os quatro resíduos. As taxas de mineralização calculadas desta forma indicam a relativa estabilidade dos diferentes resíduos, desde que não ocorra perda de $\mathrm{N}$ mineralizado por desnitrificação. Os valores absolutos, entretanto, devem ser interpretados com cautela, devido ao maior teor de $\mathrm{N}$ ter sido obtido no tratamento com adubação mineral completa (NPK), nos dois solos (Tabela 4). 


\section{ciênciarnatura UFSM, 34(1)}

Tabela 4. Teores de $\mathrm{NH}^{4+}\left(\mathrm{mg} \mathrm{kg}^{-1}\right)$, de $\mathrm{NO}^{3-}+\mathrm{NO}^{2 \cdot}\left(\mathrm{mg} \mathrm{kg}^{-1}\right)$ e $\mathrm{pH}$ em água nos solos $\mathrm{LVd} \mathrm{e}$ PVd após 88 dias de incubação.

\begin{tabular}{|c|c|c|c|c|c|c|c|c|c|}
\hline Tratamento & Dose & \multicolumn{2}{|c|}{$\mathrm{pH}^{*}$} & \multicolumn{2}{|c|}{$\mathrm{NH}^{4+}$} & \multicolumn{2}{|c|}{$\mathrm{NO}^{3-}+\mathrm{NO}^{2-}$} & \multicolumn{2}{|c|}{$\begin{array}{c}\mathrm{NH}^{4+}+\mathrm{NO}^{3}+ \\
\mathrm{NO}^{2-}\end{array}$} \\
\hline \multicolumn{10}{|c|}{ 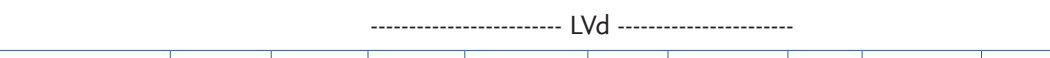 } \\
\hline Testemunha & - & 5,1 & d & 53,9 & $b$ & 29,0 & e & 82,9 & e \\
\hline NPK & - & 6,3 & c & 62,2 & $\mathrm{a}$ & 172,5 & a & 234,7 & a \\
\hline AGL & 1 & 6,7 & a & 1,2 & C & 89,4 & $d$ & 90,6 & de \\
\hline AGL & 2 & 6,5 & $a b c$ & 5,2 & c & 100,6 & c & 105,8 & $\mathrm{~cd}$ \\
\hline CERV & 1 & 6,7 & a & 1,0 & c & 91,8 & $d$ & 92,8 & de \\
\hline CERV & 2 & 6,5 & $a b c$ & 0,6 & c & 104,0 & c & 104,6 & $\mathrm{~cd}$ \\
\hline LAT & 1 & 6,6 & $a b$ & 1,6 & c & 95,5 & $d$ & 97,1 & cde \\
\hline LAT & 2 & 6,3 & c & 2,2 & c & 109,3 & c & 111,5 & c \\
\hline TEXT & 1 & 6,4 & bc & 1,2 & $\mathrm{C}$ & 97,1 & $\mathrm{~cd}$ & 98,3 & cde \\
\hline TEXT & 2 & 6,0 & d & 3,2 & c & 128,3 & $b$ & 131,5 & $b$ \\
\hline
\end{tabular}

\begin{tabular}{|c|c|c|c|c|c|c|c|c|c|}
\hline Testemunha & - & 4,6 & c & 29,8 & $d$ & 25,7 & $\mathrm{a}$ & 55,5 & e \\
\hline NPK & - & 6,0 & a & 169,8 & $\mathrm{a}$ & 30,0 & a & 199,8 & a \\
\hline AGL & 1 & 6,3 & $b$ & 49,4 & C & 31,9 & a & 81,3 & $\mathrm{~cd}$ \\
\hline AGL & 2 & 6,2 & $b$ & 51,5 & C & 30,2 & a & 81,7 & $\mathrm{~cd}$ \\
\hline CERV & 1 & 6,3 & $b$ & 50,0 & C & 26,4 & a & 76,4 & $d$ \\
\hline CERV & 2 & 6,2 & $b$ & 58,2 & C & 30,3 & a & 88,5 & $\mathrm{~cd}$ \\
\hline LAT & 1 & 6,1 & $b$ & 54,9 & C & 29,4 & a & 84,3 & $\mathrm{~cd}$ \\
\hline LAT & 2 & 6,0 & $b$ & 66,3 & C & 33,4 & a & 99,7 & bc \\
\hline TEXT & 1 & 6,2 & $b$ & 52,1 & C & 25,7 & a & 77,8 & $d$ \\
\hline TEXT & 2 & 6,0 & $b$ & 93,6 & $b$ & 26,0 & $a$ & 119,6 & $b$ \\
\hline
\end{tabular}

* Médias seguidas de mesma letra na coluna não diferem estatisticamente entre tratamentos pelo teste de Tukey $(p<0,05)$.

Conclusão

A taxa de decomposição dos resíduos nos dois solos avaliados, determinada pela liberação de $\mathrm{CO}_{2}$ e pelo acúmulo de $\mathrm{N}$ mineral no solo, aumentou na ordem: TEXT>LAT>CERV>AGL. Os resíduos orgânicos avaliados 
necessitam adubação complementar com nitrogênio mineral para serem utilizados como fonte de nutrientes para as plantas. Estudos sobre a liberação de metais pesados devido à dissociação desses resíduos no solo devem ser conduzidos para avaliar com maiores detalhes seus efeitos sobre as plantas e o ambiente.

Agradecimentos

À CAPES e ao CNPq, pelo apoio financeiro e bolsas concedidas.

\section{Referências}

CERRI, C.C.; ANDREOUX, F.; EDUARDO, B.P. Incubador para estudos sobre decomposição de material orgânico no solo. Revista Brasileira de Ciência do Solo, Viçosa, v.18, n.1, p.1-5, 1994.

EMBRAPA. Sistema brasileiro de classificação de solos. Brasília: $2^{\mathrm{a}}$ Edn. Embrapa-SPI, 2006. 306p.

GOYAL, S. MISHRA, M.M.; DHANKAR, S.s. Microbial biomass turnover and enzyme activities following the application of farmyard manure to field soils with and without previous long-term applications. Biology and Fertility of Soils, v.15, n.1, p.6o-64, 1993 .

HERNANDEZ, T. GARCIA, C.; LAX, A. Transformation of carbon and nitrogen in a calciorthid soil amended with a range of organic residues. Plant and Soil, v.105, n.1, p.205-211, 1988.

LORENZ, K.; LAL, R. Biogeochemical C and N cycles in urban soils. Environment International, v.35, n.1, p.1-8, 2009.

MAISTO, G.; MARCO, A.; NICOLA, F.; ARENA, C.; VITALE, L.; SANTO, A.v. Suitability of two types of organic wastes for the growth of sclerophyllous shrubs on limestone debris: A mesocosm trial. Science of the total environment, v.408, n.7, p.1508-1514, 2010.

MALIK, S.; BEER, M.; MEGHARAJ, M.; NAIDU, R. The use of molecular techniques to characterize the microbial communities in contaminated soil and water, Environment International, v.34, n.2, p.265-276, 2008.

METZGER, L.; YARON, в. Influence of sludge organic matter on soil physical properties. Advances in Soil Science, v.7, n.1, p.141-163, 1987. 
MOReIRA, F.M.S.; SIQUeIRA, J.o. Microbiologia e Bioquímica do Solo. 2 ed. Lavras: Editora UFLA, 2006. 729p.

ØVREÅS, L.; TORSVIK, V. Microbial diversity and community structure in two different soil agricultural communities. Microbial Ecology, v.36, n.2, p.303-315, 1998. REBAH, F.B.; PRÉVOST, D.; YEZZA, A.; TYAGI, R.D. Agro-industrial waste materials and wastewater sludge for rhizobial inoculant production: A review. Bioresource Technology, v. 98, p. 3535-3546, 2007.

SMITH, S.R. A critical review of the bioavailability and impacts of heavy metals in municipal solid waste composts compared to sewage sludge. Environment International, v.35, n.1, p.142-156, 2009.

STIJEPOVIC, M.Z.; LINKE, P. Optimal waste heat recovery and reuse in industrial zones. Energy, v. 36, p. 4019-4031, 2011.

STOTZKY, G. Microbial respiration. In: BLACK, C.A., (Ed) Methods of soil analysis Part 2: Chemical and microbiological properties. Madison: ASA, 1965. p.1551-1572. TEDeSCO, M.J. GiAnello, C.; BisSAni, C.A.; BOHnen, H.; VOlKWEiss, S.J. Análise de solo, plantas e outros materiais. 2.ed. Revisada e ampliada. Porto Alegre: Departamento de Solos da UFRGS, 1995. 174p.

ZAMORANO, M.; GRINDLAY, A.; MOLERO, E.; RODRÍGUEZ, M.I. Diagnosis and proposals for waste management in industrial areas in the service sector: case study in the metropolitan area of Granada (Spain). Journal of Cleaner Production, 2011. doi:10.1016/j.jclepro.2011.07.004.

WEI, M.S.; HUANG, K.H. Recycling and reuse of industrial wastes in Taiwan. Waste Management, v.21, p. 93-97, 2001.

Submetido em: 28/12/2010

Aceito em: $02 / 11 / 2011$ 